\title{
On MR Imaging of the Intracranial Vessel Wall
}

Can. J. Neurol. Sci. 2011; 38: 4-5

Intracranial arterial stenosis or occlusion accounts for 9-15\% of ischemic strokes in the United States, ${ }^{1}$ and an even higher proportion of strokes in Asian populations. ${ }^{2}$ Conventionally, intracranial steno-occlusive disease has been characterized using CT angiography, MR angiography, and catheter angiography. These techniques depict the effect of disease on the arterial lumen, but do not directly image the underlying pathology which most commonly involves the vessel wall. There has been burgeoning interest in MR imaging of the carotid vessel wall to differentiate between stable and unstable atherosclerotic plaque. For the (relatively smaller) intracranial arteries, 3 Tesla MR systems are much better than 1.5 Tesla MR systems, as the former provide greater signal-to-noise, enabling imaging with the spatial resolution needed to characterize the intracranial arterial wall. The MR vessel wall imaging may allow differentiation between stable and unstable intracranial atherosclerotic plaque. Furthermore, intracranial stenosis occurs secondary to a broad range of pathologies (such as atherosclerosis, vasculitis, drug-induced vasculopathy, and Moyamoya disease), with differing management, and vessel wall imaging may aid in differentiating among these.

In this issue of the Canadian Journal of Neurological Sciences, Payne et $\mathrm{al}^{3}$ present contrast-enhanced vessel wall MR imaging before and after treatment in a child with presumed primary angiitis of the CNS. Specifically, they describe a five year-old girl with an acute right lenticulotriate territory infarction, right middle cerebral artery wall thickening and enhancement, and subsequent clinical improvement and resolution of vessel wall enhancement following steroid treatment. This case illustrates the ostensible use of intracranial vessel wall imaging, and prompts several questions.

First, what is the histopathological correlate of intracranial vessel wall enhancement? That is, how does intravenouslyinjected contrast material enter the vessel wall? It is generally thought that the vasa vasora of the internal carotid arteries and vertebral arteries extend intracranially $1-2 \mathrm{~cm}$ beyond the site of dural penetration ${ }^{4}$. Older age and intracranial atherosclerosis may be associated with vasa vasora more distally, but this does not obviously explain vessel wall enhancement extending into the M2 branches of the MCA in a child. Another possibility is leaky endothelium with contrast seepage from the parent artery into the vessel wall. Perhaps higher magnetic field strength MR imaging or post-mortem histopathology, that is, greater spatial resolution, will clarify the specific location of contrast accumulation within the wall. Second, Payne et $\mathrm{al}^{3}$ have shown vessel wall enhancement in the artery supplying acutely infarcted tissue, and resolution of this enhancement over weeks. In a small study of patients with MCA stenosis and acute infarct, Klein et $\mathrm{al}^{5}$ found vessel wall enhancement up to 15 months after the acute event. Does intracranial vessel wall enhancement imply "active" disease or increased risk of future ischemic events? How does the temporal evolution of vessel wall enhancement depend on the underlying pathology and treatment? Third, Payne et $\mathrm{al}^{3}$ describe vessel wall enhancement extending from the M1 segment of the right MCA into the M2 branches. What does morphology and spatial extent of vessel wall enhancement imply? Is focal eccentric enhancement versus long segment concentric enhancement a useful distinction for diagnosing intracranial atherosclerosis versus vasculitis? In a pediatric population, with atherosclerosis excluded from a differential diagnosis, and no evidence of dissection on precontrast vessel wall imaging, is vessel wall enhancement specific for vasculitis? Intracranial vessel well enhancement may be a particularly useful finding in pediatric ischemic stroke. Is lack of wall thickening and lack of enhancement the typical pattern for Moyamoya disease ${ }^{6}$, and if so, is this true for both early and late disease? There are many important questions for future research. Payne et $\mathrm{al}^{3}$ present contrast-enhanced T1-weighted images to depict vessel wall pathology, but a variety of non-enhanced sequences may be used as well. Carotid vessel wall MR imaging has used a combination of tissue weightings (T1, T2, proton density) to identify fibrous cap, intraplaque hemorrhage, calcification, and lipid core, that is, the components of atherosclerotic plaque. In the carotid arteries, there is evidence that intraplaque high signal intensity corresponds with recent intraplaque hemorrhage, and indicates increased risk of future transient ischemic attack or stroke. ${ }^{7}$ In a post-mortem study, MCA plaques associated with infarction had a higher prevalence of neovascularisation and intraplaque hemorrhage than plaques not associated with infarction. ${ }^{8}$ Ryu et al demonstrated focal areas with differing MR signal intensities within MCA plaques in vivo, but without histopathological correlation. ${ }^{9}$ High resolution non-enhanced T1-weighted images are certainly helpful for the diagnosis of intracranial arterial dissection.

Basic implementation of intracranial vessel wall imaging is not difficult, and may be accomplished using standard MR pulse sequences. This is more easily accomplished on 3 Tesla MR systems due to higher intrinsic signal-to-noise than on 1.5 Tesla systems. For an institute interested in using this technique, a starting point might be to run a T1-weighted 2D FLAIR fast spin echo sequence (slice thickness $3 \mathrm{~mm}$, matrix size 512 x 512) in plane and perpendicular to the vessel of interest, before and after intravenous administration of gadolinium.

Unlike carotid artery disease, for which endarterectomy specimens are available, intracranial vessel wall specimens are rare, and progress may largely depend on detailed correlation of serial vessel wall imaging with clinical and laboratory findings. Further development of dedicated MR pulse sequences, and further study of the breadth of patients with intracranial disease will almost certainly lead to both a better understanding of those 
diseases affecting the intracranial arteries, and a clinically important diagnostic tool.

Daniel M. Mandell, Manohar Shroff University of Toronto Toronto, Ontario, Canada

\section{REFERENCES}

1. White H, Boden-Albala B, Wang C, et al. Ischemic stroke subtype incidence among whites, blacks, and Hispanics: the Northern Manhattan Study. Circulation. 2005;111:1327-31.

2. Suri MFK, Johnston SC. Epidemiology of intracranial stenosis. J Neuroimaging. 2009;19 Suppl 1:11S-16.

3. Payne ET, Wei X, Kirton A. Reversible wall enhancement in pediatric cerebral arteriopathy. Can J Neuro Sci. 2011;38(1): $139-40$.
4. Takaba M, Endo S, Kurimoto M, et al. Vasa vasorum of the intracranial arteries. Acta Neurochirurgica. 1998;140:411-16.

5. Klein IF, Lavallee PC, Touboul PJ, et al. In vivo middle cerebral artery plaque imaging by high-resolution MRI. Neurology. 2006; 672:327-9.

6. Swartz RH, Bhuta SS, Farb RI, et al. Intracranial arterial wall imaging using high-resolution 3-tesla contrast-enhanced MRI. Neurology. 2009;72:627-34.

7. Altaf N, Daniels L, Morgan PS, et al. Detection of intraplaque hemorrhage by magnetic resonance imaging in symptomatic patients with mild to moderate carotid stenosis predicts recurrent neurological events. J Vasc Surg. 2008;47:337-42.

8. Chen XY, Wong KS, Lam WW, et al. Middle cerebral artery atherosclerosis: histological comparison between plaques associated with and not associated with infarct in a postmortem study. Cerebrovasc Dis. 2008;25:74-80.

9. Ryu CW, Jahng G, Kim EJ, et al. High resolution wall and lumen MRI of the middle cerebral arteries at 3 Tesla. Cerebrovasc Dis. 2009;27:433-42. 